\title{
Board 10: Work in Progress: A Blended Model for a Biomaterials Course Improves Student Learning and Allows for Enhanced Content
}

\section{Dr. John P Puccinelli, University of Wisconsin, Madison}

Dr. Puccinelli is the Associate Chair of the Undergraduate Program in the Department of Biomedical Engineering. He began here as student near the start of the UW-BME program and earned his BS, MS, and $\mathrm{PhD}$ in BME. He is interested in hands-on instruction - teaching and developing courses related to biomaterials and tissue engineering, as well as design. 


\section{Work in Progress: A blended model for a biomaterials course improves student learning and allows for enhanced content}

The field of biomaterials, while still rapidly growing, is rooted in foundational materials science and immunology. Here, we require a 3 credit, introductory biomaterials course ( 115 students) called "Biological Interactions with Materials" and employ Ratner's "Biomaterials Science: An Introduction to Materials in Medicine" textbook. Over the last 20 years, the text itself has grown from 484 pages in the first edition to 864 pages in the second edition and to now 1573 pages in the latest, third edition. Covering this volume of material and detail in a one semester introductory course has become increasing challenging. To accommodate this growing body of knowledge, as well as growth in our student population, we went from a completely lecturebased course to a blended model using well documented active learning strategies [1-4]. The format we employed utilizes online lectures covering the foundational materials science, immunology, and traditional application of biomaterials. The in-class time was then repurposed for three activities: 1. lectures on the latest research and applications in the field, 2. group discussions around case-studies, and 3. hands-on laboratories. While the online material received mixed reviews, the repurposed activities were appreciated and improvements were seen in final exam scores.

\section{Introduction}

Our biomaterials course had been developed as a purely lecture-based course that complimented readings from Ratner's textbook. The course was divided into three modules: 1 . Classic synthetic biomaterials, 2: biological based materials, proteins, cell and tissue, and 3. Host response to materials. Each module concluded with an exam with the third exam requiring past knowledge from the whole course, but heavily weighted on the new content. Throughout the semester, within the two weekly 75 min lectures, new material such as current events surround biomaterials, case-studies and research topics would be interjected, but unfortunately only briefly given time constraints. Past work by others successfully employed active learning in a biomaterials course [5]. Additionally, we wished to provide a hands-on component within this course to further strengthen concepts [6]. In order to do so effectively, we blended our biomaterials course as outlined below.

\section{Structure}

The new course structure was similar in nature to our traditional lecture schedule where we divided the course into the same three ( five week long) modules with three exams. As describe in detail below, core content was converted to sets of online lectures. This opened the in classperiods to enhance and supplement the content with active learning sessions and novel biomaterial applications. The in-class periods for each module consisted of the following: 1. A module overview which included research related to the module and summary of the textbook, 2. A research lecture by a faculty member performing research that aligned with the module, 3 . a case-study from the textbook that helped to summarize a clinical application of the module, 4 . a hands-on laboratory exercise, 5. a review session (previously also held during the lecture only offerings), and 6. the exam. As the online content would build each week, as did the depth of the in-class activities. 


\section{Online Lectures}

We had previously recorded two years of the biomaterials course lectures using our Universities lecture capture system. With this core biomaterials content in hand, the videos were carefully selected, updated, and edited down to eliminate course logistics content, questions posed to class and from the class, the brief discussions and current events previously presented in the lecture. Each video was then 20-50 minutes long covering one major course topic (about one hour of online content was assigned each week for a total of six-seven topics per module). We then developed a quiz for each topic video consisting of five questions where three-four questions were directly taken from key points in the lecture and one-two questions were more challenging requiring assimilation from past material and/or the textbook readings. As the online videos would have resulted in a substantially larger workload for the course, we employed a replacement model and eliminated three meeting times early in each module where extended office hours were held during the class period.

\section{Case-study Discussions}

The second in-class session of each module applied early concepts and introduced later concepts through an interactive discussion around a case-study. The examples came from readings available in the back of the textbook in the section titled "Practical Aspects of Biomaterials" mixed with related current events. Examples included: material design considerations, regulatory constraints, and ethical dilemmas (i.e those surrounding failed implants). An overview was presented in the first 10 min of the case-study session. For the next 20-30 minutes students worked in groups on a worksheet while the instructors and TAs circulated around the room. The last 30-40 min was used to report out the groups' findings. With such a large class, the worksheet questions were either divided between groups in the class or during one case-study the room was split in two to debate on the side of the medical device company or on the side of the patient who had a failed implant. Students were held accountable for the material via attendance and participation in their group/whole class discussion (a tally system was used by the instructors and TAs) where they received credit/no credit for the activity. Worksheets were not collected, however, questions based on the case-study discussions appeared on the exams.

\section{Research Lectures and Presentations}

The third in-class session of each module consisted of a faculty-led research lecture. Discussions were held with the three guest lecturers to develop an appropriate lecture that focused primarily on cutting edge research being performed in their lab that tied in directly with the learning objectives of their module. Additional applications were presented at the end of the course in a few 30 min biomaterial presentations from the handful of graduate students in the course. Throughout the semester, the graduate students performed literature research projects which three check points in which specific parts of the project were assigned. These check-points coincided with the hands-on laboratories performed by the undergraduate students (the majority of the class).

\section{Hands-on Activities}

The content of each module ended with a hands-on laboratory for the undergraduates that was held in our dedicated biomaterials and biomechanics teaching laboratory. The large class was split into two groups. One group attended a review session while the other group divided into teams of fourfive students for the lab (the groups switch for the following class period). Lab activities were limited to the $75 \mathrm{~min}$ class period. The three labs were: 1. fabricating materials and investing 
structural functional relationships (mechanical properties) using alginate, 2. material modification and surface properties by cross-linking gelatin and measure contact angle on material coated glass slides in the presence and absence of adsorbed proteins, and 3. investigating hydrogel swelling and degradation in a biomimetic environment. Teams submitted a team lab report for assessment.

\section{Results}

Over four years we administered near identical multiple choice and true/false exams at the end of the third module (which assimilates content across the course). We compared undergraduate scores from two years with live lectures/no active learning-'pre' and two years with online lectures/active learning-'post' for identical questions (those not associated specifically with the guest lectures for example). The results, shown in Figure 1, demonstrate that students in the active learning version performed by half a grade better on average and a reduction in lower-end scores, the 'tail' was seen. Student population demographics were similar based upon consistency in departmental admissions, however, we did not control for this variable.

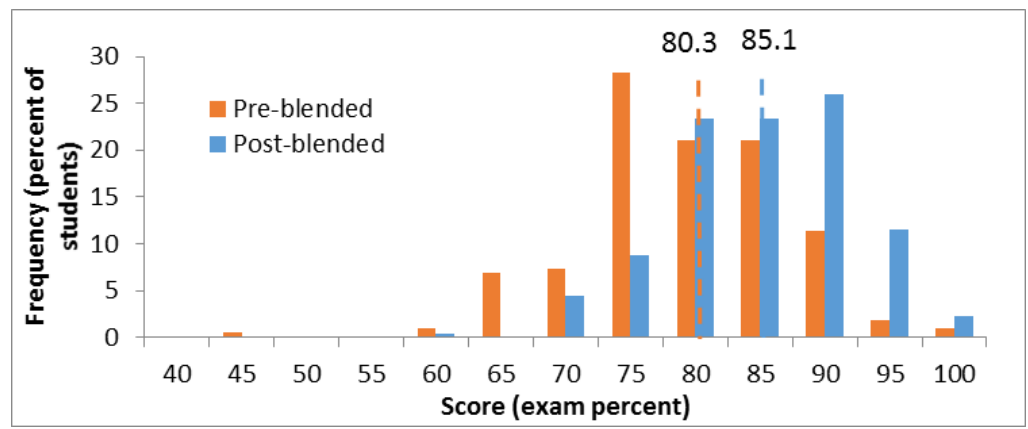

Figure 1. Improved learning shown in the last exam scores for identical questions over four years (two pre- and two post-blending). Bins are shown as equal to or greater than the numerical value. (pre $n=219$ and post $n=227$ )

Despite the positive gains in student performance, the students were discontent with the online videos (compared to when they were presented live) based on end of the semester teaching evaluation comments. Additionally, the instructor rating was reduced (though still acceptable) and comments described a preference for in person lectures, the ability to gauge the instructor's enthusiasm and the ability to ask more questions directly related to the content in real-time.

Positive comments included "The course structure was really well set up with the online lectures, exams, and case studies. I enjoyed how dynamic the learning was. Sometimes it seemed a little fast paced, but overall it was very good and very useful!" and "I liked the case studies. They're a good way to make you think about all the things that have to go into a medical device." The labs and guest lectures were also well-received with numerous positive comments.

\section{Conclusion}

The structure of the course utilized online lectures and the textbook to build the foundation, base knowledge and core content of the course. The case-study reinforced the content through a reallife medical application and discussions. The research lectures presented how the material can be used a novel way and further reinforced the core content. Finally, the labs visually demonstrated key concepts. The mixed reviews of the online lecture style has prompted us reduce the online content significantly while maintaining the positive aspects of the course going forward. Future assessment includes identifying learning-outcome gains by looking at specific exam questions, a direct survey about each aspect of the course and engagement with instructors of courses for which this is a requisite to assess long-term gains and preparation. 


\section{References}

1. P. Moskal, C. Dziuban, and J. Hartman, "Blended Learning: A Dangerous Idea?" Internet and Higher Education, 18(C), pp. 15-23, 2013.

2. C. Twigg, "Improving Learning and Reducing Costs: New Models for Online Learning," EDUCAUSE Review, pp 28-37, Sept/Oct 2003.

3. C. Bonwell and T. Sutherland, "The active learning continuum: Choosing activities to engage students in the classroom." New directions for teaching and learning, p3-16, 1996.

4. J. Faust and D. Paulson, "Active Learning in the College Classroom." Journal on Excellence in College Teaching, pp 3-24, 1998.

5. W. Guilford and M. Lawrence, "A Course in Biomaterials Taught Using the Socratic Method," ASEE Annual Conference \& Exposition, Indianapolis, Indiana, 2014.

6. J. Vernengo, "Active and Cooperative Learning Activities for an Introductory Biomaterials

Course," ASEE Annual Conference \& Exposition, Vancouver, BC, 2011. 